\title{
GLOBAL STABILITY OF DISCRETE-TIME COMPETITIVE POPULATION MODELS
}

\begin{abstract}
We develop practical tests for the global asymptotic stability of interior fixed points for discrete-time competitive population models. Our method constitutes the extension to maps of the Split Lyapunov method developed for differential equations. We give ecologically-motivated sufficient conditions for global stability of an interior fixed point of a map of Kolmogorov form. We introduce the concept of a principal reproductive mode, which is linked to a normal at the interior fixed point of a hypersurface of vanishing weighted-average growth. Our method is applied to establish new global stability results for 3-species competitive systems of May-Leonard type, where previously only parameter values for local stability was known.
\end{abstract}

\author{
Stephen Baigent \\ Department of Mathematics, UCL, Gower Street, \\ London WC1E 6BT, UK \\ ZHANYUAN HOU \\ School of Computing and Digital Media, London Metropolitan University, \\ 166-220 Holloway Road, London N7 8DB, UK
}

\section{INTRODUCTION}

[Note This paper was published in Journal of Difference Equations and Applications, Volume 23 (2017), pp. ?-? (doi:10.1080/10236198.2017.1333116). Published online in May 2017]

Here we describe a general test for global stability of a coexistence state that can be successfully applied to a wide range of discrete-time competitive population models, and we apply the test to some standard models, including some whose global stability properties are unresolved.

Thus, let us assume that the model has $N$ interacting species with population densities $x_{i} \geq 0$ for $i \in I_{N}:=\{1,2, \ldots, N\}$ and write $x=\left(x_{1}, \ldots, x_{N}\right)^{\top}$ (a column vector). We set $\mathcal{C}=\mathbb{R}_{+}^{N}$ for the nonnegative first orthant, where $\mathbb{R}_{+}=[0, \infty)$. We use the natural numbers $\mathbb{N}=\{0,1, \ldots\}$ for the model time units. Let $f=\left(f_{1}, \ldots, f_{N}\right)^{\top}: \mathcal{C} \rightarrow \mathbb{R}^{N}$ be bounded and continuous in each component. Then the Kolmogorov model that we consider is

$$
x_{i}(t+1)=T_{i}(x(t))=x_{i}(t) f_{i}(x(t)), i \in I_{N}, \quad t \in \mathbb{N} .
$$

1991 Mathematics Subject Classification. Primary: 39A11; Secondary: 92D25.

Key words and phrases. competitive Kolmogorov maps; global stability; Leslie-Gower; Ricker; principal reproductive mode. 
For each initial state $x \in \mathcal{C}$, equation (1) generates a forward orbit $O^{+}(x)=\{x(t)\}_{t \in \mathbb{N}}$ with $x(t)$ denoting the population state reached from the initial state $x(0)=x$ after $t$ time steps.

As with many studies of the global dynamics of population models, our approach is based upon a Lyapunov function. In fact, the Lyapunov function that we choose has been previously used as an entropic asymmetric distance function in continuous-time population models $[11,31]$, and also as an average Lyapunov function in the study of permanence of population models $[10,12,13]$. Our method extends the average Lyapunov function approach, a technique which is usually applied only over a finite time interval, and extends it over all forward time to extract long term properties of a permanent system.

The framework that we use is as follows. The model (1) is assumed to be competitive (see definition 1.5) and to have a unique interior fixed point: $p \in \mathcal{C}=(0, \infty)^{N}$. Let $v$ be a given positive vector and $V(X)=\prod_{i=1}^{N} X_{i}^{v_{i}}$ with $X=\left(X_{1}, \ldots, X_{N}\right)^{T}$; this is the Lyapunov function we employ that has also been widely used as an average Lyapunov function $[10,12,13]$ for showing permanence. The vector $v$ is similar to that identified as Reproductive Value in classic Leslie matrix models (e.g. [3, 29]) in that it is a left eigenvector associated with the dominant eigenvalue of an irreducible nonnegative matrix. Then for $x(0)=x$, and $x(t)=T^{t}(x) \in O^{+}(x)$,

$$
V(x(t))=\psi(t, x) V(x),
$$

where $\psi(t, x)=\exp \left(v^{\top} \sum_{k=0}^{t-1} \ln (f(x(k)))\right) . \quad\left(\right.$ Here $\ln \left(x_{1}, \ldots, x_{n}\right)=\left(\ln \left(x_{1}\right), \ldots, \ln x_{n}\right)^{\top}$ for $x \in \mathcal{C})$. Notice that $\psi(t, x)$ is nondecreasing along the forward orbit $O^{+}(x)$ when the sum

$$
\Phi(t, x)=\sum_{k=0}^{t-1} v^{\top} \ln (f(x(k)))
$$

is nondecreasing. Thus a necessary and sufficient condition for $V$ to be nondecreasing (i.e. a Lyapunov function) along $O^{+}(x)$ is that $v^{\top} \ln (f(x(t))) \geq 0$ for each $t \in \mathbb{N}$. An important feature of the expression $\Phi(t, x)$, is the weighting in the sum by the vector $v$, and an ecologically-motivated choice of $v$ is the key to the method's success, as we explain later.

The key property that our models satisfy is:

Property 1: Given any $x \in \mathcal{C}^{\circ}$ and $O^{+}(x)=\{x(t)\}_{t \in \mathbb{N}}$ there exists a $t^{\prime}$ (which may depend on $x$ ) such that $v^{\top} \ln (f(x(t))) \geq 0$ for all $t \geq t^{\prime}$.

Property 1 states that an interior orbit eventually enters a certain region of phase space where the Lyapunov function $V$ then is nondecreasing, and Lyapunov theory of LaSalle 
applies [21]. A significant part of this paper is developing sufficient conditions for Property 1 above to hold. To this end we introduce

Definition 1.1. The vector $\alpha=D[p]^{-1} v$ will be named the principal reproductive mode.

Definition 1.2. With $v=D[p] \alpha$, the function $\varphi(X)=\alpha^{\top} D[p] \ln f(X)$ appearing in Property 1 will be named the principal component of the reproductive rate.

(Here and elsewhere $D[x]$ is the diagonal matrix with diagonal elements $x_{1}, \ldots, x_{n}$.) The principal component of the reproductive rate is a scalar that provides a measure of the component of the per-capita growth of the whole population in the direction of the dominant mode $\alpha$.

From a practical point of view, our results express the following principle:

For a bounded population model with unique coexistence state $p$, if the principal component of the reproductive rate is eventually positive, the population converges to $p$.

A summary of the geometry of our method is shown in figure 1 .

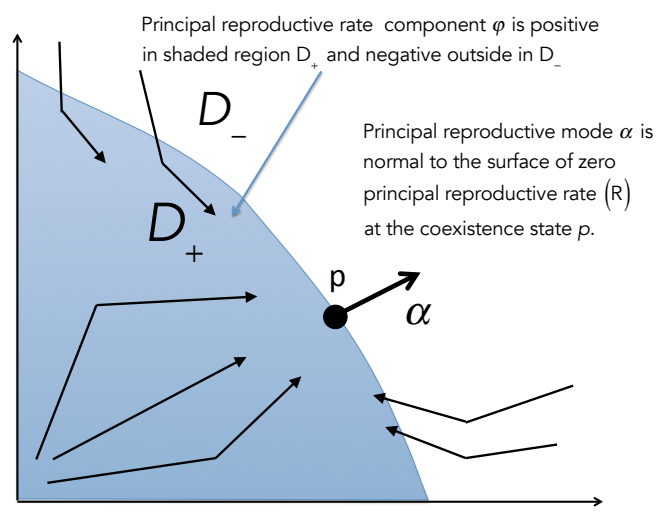

FiguRE 1. A summary of our method. All trajectories eventually end up in the shaded region $\mathcal{D}_{+}$where the principal reproductive rate component is positive, and then converge to the coexistence fixed point $p$. The surface of zero principal reproductive rate (the boundary of $\mathcal{D}_{+}$in $C$ ) has an outward normal at $p$ that points along the vector $\alpha$, the principal reproductive mode.

As practical demonstrations, we apply our methods to establish global stability in the case of 3 species for two well-known competitive models, namely the Leslie-Gower model and 
the Ricker model. These take the form

$$
\begin{aligned}
x_{i}^{\prime} & =T_{i}(x)=\frac{b x_{i}}{1+(\mathcal{A} x)_{i}} \quad(i=1,2,3) \quad \text { (Leslie-Gower) } \\
x_{i}^{\prime} & =T_{i}(x)=x_{i} \exp \left(r\left(1-(\mathcal{A} x)_{i}\right)\right) \quad(i=1,2,3) \quad \text { (Ricker) }
\end{aligned}
$$

where $x_{i}, x_{i}^{\prime}$ are the current and next generation population densities of species $i$ respectively, $r, b>0$ constants, $\mathcal{A}$ is a positive $3 \times 3$ matrix

$$
\mathcal{A}=\left(\begin{array}{lll}
1 & \alpha & \beta \\
\beta & 1 & \alpha \\
\alpha & \beta & 1
\end{array}\right),
$$

and $\alpha, \beta>0$ reflect the strength of competition.

Note that for the Leslie-Gower model, the map $T$ is injective on the first orthant. However, the Ricker map is not injective on the first orthant, but it is on the forward invariant compact set $T \mathcal{C}$.

For these Leslie-Gower and Ricker we establish:

Theorem 1.3 ((Global stability for the 3-species May-Leonard Leslie-Gower model)). Suppose that $b>1,0<\alpha+\beta<2$ and either

$$
b\left(4 \alpha^{2}+4 \beta^{2}-4 \alpha-4 \beta-\alpha \beta+1\right)<3\left(\alpha^{2}+\beta^{2}-\alpha-\beta-\alpha \beta+1\right),
$$

or

$$
3 b(1-2 \alpha-2 \beta+3 \alpha \beta) \geq 5(1-\alpha-\beta)+7 \alpha \beta-\alpha^{2}-\beta^{2} .
$$

Then the Leslie-Gower model has a unique fixed point that is globally asymptotically stable in the interior of the first orthant.

Theorem 1.4 ((Global stability for the 3-species Ricker model)).

Suppose that $r \in(0,1), \alpha+\beta<2$ and either

$$
3 r\left(1+\alpha^{2}+\beta^{2}-\alpha-\beta-\alpha \beta\right)<(2-\alpha-\beta)(1+\alpha+\beta),
$$

or

$$
r\left(5-\alpha^{2}-\beta^{2}-5(\alpha+\beta)+7 \alpha \beta\right) \geq(2-\alpha-\beta)(1+\alpha+\beta) .
$$

Then the Ricker model has a unique fixed point that is globally asymptotically stable in the interior of the first orthant.

Local asymptotic stability of interior fixed points in these models (indeed their $N$-species versions) were studied by Roeger in a series of papers [22, 23, 24] and recently global stability of the $N$-species periodic Leslie-Gower model was studied [26] using contraction mapping techniques (see also the planar Ricker in [28]). An advantage of our approach is that it yields a detailed description of parameter values sufficient for global stability. A disadvantage over [26] is that our method cannot directly deal with periodic orbits. However, it might be possible to overcome this shortcoming by working with powers of the $\operatorname{map} T$. 
Global stability results were obtained by Smith for planar competitive maps [30] by exploiting the monotonicity of these maps with respect to a non-standard orthant ordering. The planar Leslie-Gower model and Ricker model (for some parameter values) fit into this framework. Since there does not appear to be a suitable ordering to render 3-species discrete-time competitive models monotone, it seems likely that a similar approach will not lend itself to general discrete-time competitive models and alternatives need to be found. In a little-cited 1977 paper, Fisher and Goh [4] present a Lyapnuov function approach to global stability of discrete-time population models. Here we develop a similar approach, but with a different Lyapunov function.

Geometrical approaches to (1) have been developed by Franke and Yakubu in a series of papers $[5,7,8]$, and also by Kon in [19]. Although focussing on exclusion principles in [5] Franke and Yakubu study global attractors of 2-species discrete-time competitive models. In a similar, but distinct geometrical approach, Kon also studied permanence and mutual exclusion principles in discrete-time competitive population models [18, 19, 20], and in [19] Kon presents a geometrically-derived exclusion principle based upon the convexity or concavity of the per-capita growth rate functions.

Recently Sacker [26] used dynamical reduction to demonstrate global stability in a multispecies periodic Leslie-Gower model. Theorem 3.5 in [26] confirms that in the autonomous case, the Leslie-Gower model with sufficiently weak competition (to ensure that the mapping that he constructs is a contraction) globally attracts the interior of the first orthant. Other models, including those of Ricker type are also considered in an earlier paper [28].

The main objective of this paper is to link the geometrical flavour of the work of Franke, Yakube, Kon and others, with the method of the Split Lyapunov function for continuoustime Kolmogorov systems developed by E. C. Zeeman and M. L. Zeeman in [32], Hou and Baigent $[1,14,15]$.

1.1. Notation and standing assumptions. Let $\mathcal{X}$ be a metric space and $T: \mathcal{X} \rightarrow \mathcal{X}$ be continuous. If $T$ is also continuously differentiable, we denote by $D T$ its derivative. For a given $x \in \mathcal{X}$ one typically studies the sequence $O^{+}(x):=\left\{T^{t}(x): t \in \mathbb{N}\right\}(\mathbb{N}=\{0,1,2, \ldots\})$ which is referred to as the forward orbit of $T$ through $x$. A set $\mathcal{A} \subset \mathcal{X}$ is forward $T$-invariant if $T(\mathcal{A}) \subseteq \mathcal{A}$ and $T$-invariant if $T(\mathcal{A})=\mathcal{A}$. The set of fixed points of $T$ is $\operatorname{Fix}(T)=\{x \in$ $\mathcal{X}: T(x)=x\}$. For a given set $\mathcal{W} \subset \mathcal{X}$, let $\omega(\mathcal{W}):=\bigcap_{n=0}^{\infty}\left(\overline{\bigcup_{k=n}^{\infty} T^{k}(\mathcal{W})}\right)$ denote the omega limit set of $\mathcal{W}$. When $O^{+}(x)$ has compact closure in $\mathcal{X}, \omega(x)=\omega(\{x\})$ is a nonempty, compact and invariant set. We call an invariant set $\mathcal{A} \subset \mathcal{X}$ an attractor if there exists an open $\mathcal{U} \supset \mathcal{A}$ for which $\omega(\mathcal{U})=\mathcal{A}$. By an absorbing set we mean a set $B \subset \mathbb{R}^{N}$ such that for any bounded set $\Theta \subset \mathbb{R}^{N}$ there is a $t_{\Theta} \in \mathbb{N}$ such that $T^{t} \Theta \subset B$ for all $t \geq t_{\Theta}$. $T$ is called dissipative if it has a compact absorbing set $B$.

Let $D \subset \mathbb{R}^{N}$. Recall the following partial ordering for vectors $u \in \mathbb{R}^{N}$ : (a) $u \leq v$ if $u_{i} \leq v_{i}$ for $i \in I_{N}$, (b) $u<v$ if $u \leq v$ but $u \neq v$, and (c) $u \ll v$ if $v-u \in \mathcal{C}$. Similar orderings 
are used for matrices, so that, for example, for a given matrix $\mathcal{A}, \mathcal{A}>0$ means that each element of $\mathcal{A}$ is nonnegative, and at least one element is positive.

Definition 1.5. A map $T: D \rightarrow \mathbb{R}^{N}$ is competitive (strongly competitive) if $T(x)<$ $T(y) \Rightarrow x<(\ll) y$ for each $x, y \in D$.

A sufficient condition for $T$ to be strongly competitive on a set $D$ is that $D T^{-1}(x) \gg 0$ for $x \in D$.

Here we only study stability of coexistence states. The stability of boundary fixed points have been studied in $[6,7,8,9,18,19,20,25]$.

\section{ForWARD INVARIANCE OF SETS UNDER MAPS}

In discrete-time systems, even if the map $S$ maps all boundary points into $\mathcal{B}$, then some points in $\stackrel{\circ}{\mathcal{B}}$ may be mapped outside of $\mathcal{B}$, and then $\mathcal{B}$ is not forward invariant.

To obtain forward invariance based upon movement of boundary points, which is typically much more practical to implement, we can appeal to the recent result of Sacker [27] which shows that openness of the map is what is missing:

Theorem 2.1 ((Sacker)). Let $\mathcal{D} \subset \mathbb{R}^{N}$ be a bounded subset and $S: \overline{\mathcal{D}} \rightarrow \mathbb{R}^{N}$ be continuous.

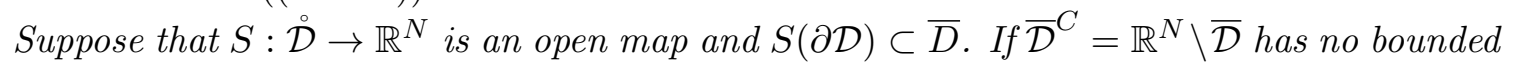
components then $S(\overline{\mathcal{D}}) \subset \overline{\mathcal{D}}$.

(A map is open if it maps open sets to open sets). When $S$ is continuously differentiable, a sufficient condition for $S$ to be an open map is that it is a local homeomorphism on $\stackrel{\circ}{D}$, which is the case when $\operatorname{det} D S$ does not vanish on $\stackrel{\circ}{D}$.

Note that the openness condition can be restrictive. Our next result removes this restriction and the boundedness requirement of $\mathcal{D}$, but at the same time has its own restrictive condition that the set $\mathcal{D}$ is connected.

Theorem 2.2. Let $\mathcal{D} \subset \mathbb{R}^{N}$ with $\mathcal{\mathcal { D }}$ a connected set and $\overline{\mathcal{D}}=\overline{\mathcal{D}}$. Let $S: \overline{\mathcal{D}} \rightarrow \mathbb{R}^{N}$ be continuous. Assume that there is a $p_{0} \in \mathcal{D}^{\circ}$ such that $S\left(p_{0}\right) \in \dot{\mathcal{D}}$ and no interior points of $\mathcal{D}$ are mapped to the boundary of $\mathcal{D}$ under $S:$

$$
\forall x \in \partial \mathcal{D},\{y \in \mathcal{D}: S(y)=x\}=\emptyset .
$$

Then $S(\overline{\mathcal{D}}) \subset \overline{\mathcal{D}}$ and $S(\mathcal{D}) \subset \mathcal{D}$.

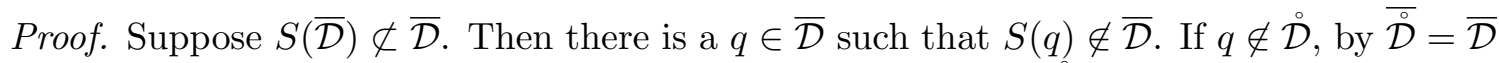
and the continuity of $S$, we can always choose a point $q^{\prime} \in \mathcal{D}$ such that both $\left\|q^{\prime}-q\right\|$ and $\left\|S\left(q^{\prime}\right)-S(q)\right\|$ are sufficiently small so that $S\left(q^{\prime}\right) \notin \overline{\mathcal{D}}$. Without loss of generality, we assume that $q \in \mathcal{D}$ with $S(q) \notin \overline{\mathcal{D}}$. Since $\mathcal{\mathcal { D }}$ is connected, there is a continuous curve 
$c \subset \mathcal{D}$ connecting $p_{0}$ to $q$. Since $S\left(p_{0}\right) \in \mathcal{D}$ but $S(q) \notin \overline{\mathcal{D}}$, by continuity of $c$ and $S$ there is $r \in c \subset \mathcal{D}$ such that $S(r) \in \partial \mathcal{D}$. This contradicts $(3)$. Therefore, we must have $S(\overline{\mathcal{D}}) \subset \overline{\mathcal{D}}$.

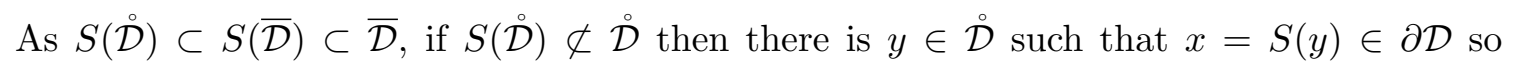
$S^{-1}(x) \cap \dot{\mathcal{D}} \neq \emptyset$, a contradiction to $(3)$. Hence, we also have $S(\mathcal{D}) \subset \mathcal{D}$.

Both Theorem 2.1 and Theorem 2.2 will play a part in the results developed below.

\section{Global attraction of Interior Fixed POINTS}

The following lemma, a version of LaSalle's invariance theorem [21], provides a test for global convergence to an interior fixed point $p$. It does not require the results of section 2 for application. It is possible to use Theorem 6.3 of [21] directly for the following result; however, some preparation is necessary to allow for the application of Theorem 6.3 (the problem lies with the boundary $\partial \mathcal{C}$ ), and we believe a more direct proof by modifying proofs of Theorem 6.3 is more natural.

Lemma 3.1. For system (1) with $f$ continuous, assume that the following conditions hold.

(i) There is a compact set $\mathcal{K} \subset \mathcal{C}$ such that

$$
\forall x(0)=x \in \mathcal{C}, \exists t_{1} \geq 0, \forall t \geq t_{1}, x(t) \in \mathcal{K} .
$$

(ii) There is a $v \in \mathcal{C}$ such that $\forall x \in \mathcal{K}$ with $x(0)=x$, either

$$
\forall t \in \mathbb{N}, \varphi(x(t))=v^{\top} \ln f(x(t)) \geq 0
$$

or

$$
\forall t \in \mathbb{N}, \varphi(x(t))=v^{\top} \ln f(x(t)) \leq 0,
$$

and $\{X \in \partial \mathcal{C}: \varphi(X) \leq 0\}$ contains no invariant set.

Then for each $x \in \mathcal{C}$, there is a constant $c>0$ such that

$$
\omega(x) \subseteq \mathcal{K} \cap \varphi^{-1}(0) \cap V^{-1}(c) .
$$

In addition, if $T$ has an interior fixed point $p$, and $X \in \mathcal{K} \cap\left(\varphi^{-1}(0) \backslash\{p\}\right)$ implies $T(X) \notin$ $\varphi^{-1}(0) \backslash\{p\}$, then $p$ is globally attracting.

Proof. Since $\mathcal{K}$ is absorbing we need only consider initial points $x \in \mathcal{K}$. Define $V(X)=$ $\prod_{i=1}^{N} X_{i}^{v_{i}}, \psi(X)=V(f(X))$. Take $x \in \mathcal{C} \cap \mathcal{K}$ and write $x(t)=T^{t}(x), V_{t}=V(x(t))$ and $\psi_{t}=\psi(x(t))$. We have $V_{0}=V(x(0))=V(x), \psi_{0}=\psi(x(0))=\psi(x)$ and $V_{t+1}=\psi_{t} V_{t}$ and $V_{t}=\exp \left(\sum_{k=0}^{t-1} \varphi\left(x_{k}\right)\right) V_{0}$. Suppose (4) holds. Then $V_{t}$ is monotone nondecreasing with $t$ and bounded by (i). Therefore, $\lim _{t \rightarrow \infty} V_{t}=c$ for some $c>0$. Let $y \in \omega(x)$ so that $x_{t_{k}} \rightarrow y$ as $k \rightarrow \infty$ for some sequence $t_{k} \rightarrow \infty$ as $k \rightarrow \infty$. Since $\lim _{k \rightarrow \infty} V_{t_{k}}=c$, continuity 
of $V$ shows that $V(y)=c$. This shows that $\omega(x) \subset V^{-1}(c)$. Now let $O^{+}(y)$ be the forward orbit through $y$. Since $\omega(x)$ is invariant, $c=V(y(t+1))=\psi(y(t)) V(y(t))=\psi(y(t)) c$, so that since $c>0, \psi(y(t))=1$ for each $t \in \mathbb{N}$. This shows that $y \in \varphi^{-1}(0)$ and hence $\omega(x) \subset \varphi^{-1}(0)$. Thus $\omega(x) \subset \varphi^{-1}(0) \cap V^{-1}(c)$.

Alternatively suppose (5) holds. Now $V_{t}$ is monotone nonincreasing in $t$ so there is a $c \geq 0$ such that $\lim _{t \rightarrow \infty} V_{t}=c$ and so $\omega(x) \subset V^{-1}(c)$. If $c=0$, then by the definition of $V$, $V^{-1}(c)=\partial \mathcal{C}$ so $\omega(x) \subset \partial \mathcal{C}$ and $\psi(y) \leq 1$, i.e. $\varphi(y) \leq 0$ for all $y \in \omega(x)$. This leads to $\omega(x) \subset\{x \in \partial \mathcal{C}: \varphi(x) \leq 0\}$, a contradiction to condition (ii) as $\omega(x)$ is invariant. Therefore, we must have $c>0$. Then by the same reasoning as before, we have $\omega(x) \subset$ $\mathcal{K} \cap \varphi^{-1}(0) \cap V^{-1}(c)$.

Now suppose $y \in \mathcal{K} \cap\left(\varphi^{-1}(0) \backslash\{p\}\right)$ implies $T(y) \notin \varphi^{-1}(0) \backslash\{p\}$. Assume $\omega(x) \neq\{p\}$ and let $y \in \omega(x) \backslash\{p\}$. Then, as $\omega(x) \subset \varphi^{-1}(0) \cap V^{-1}(c) \subset \varphi^{-1}(0)$, we have $y \in \mathcal{K} \cap\left(\varphi^{-1}(0) \backslash\{p\}\right)$ so $T(y) \notin \varphi^{-1}(0) \backslash\{p\}$. On the other hand, however, the invariance of $\omega(x)$ ensures that $T(y) \in \omega(x) \subset \varphi^{-1}(0)$. Thus, we must have $T(y)=p$. This shows that $\omega(x)=T(\omega(x))=$ $\{p\}$, a contradiction to our supposition. Therefore, $\omega(x)=\{p\}$.

\section{Split-Lyapunov Stability of interior FiXed POINTS}

From now we make some additional assumptions regarding the map $T$ :

(1) Assumption A1 The system (1) is dissipative with compact absorbing set $\mathcal{K} \subset \mathcal{C}$;

(2) Assumption A2 $f: \mathcal{C} \rightarrow \mathcal{C}$ is a continuously differentiable competitive map on a neighbourhood of $\mathcal{K}$;

(3) Assumption A3 There is a unique interior fixed point $p$ of $T$ and $D T(p)^{-1} \gg 0$.

Up to now, and in particular for lemma 3.1, we have not specified how the vector $v$ that is used to construct the Lyapunov function $V$ is to be chosen. Now we turn to the discretetime version of the Split Lyapunov method introduced for competitive Lotka-Volterra differential equations in [32] and developed further for general Lotka-Volterra systems in $[1,14]$ and for general Kolmogorov differential equations in [15].

In the following, we will repeatedly use the following sets.

$$
\begin{aligned}
& \mathcal{D}_{+}=\{X \in \mathcal{C}: \varphi(X)>0\}, \\
& \mathcal{D}_{-}=\{X \in \mathcal{C}: \varphi(X)<0\}, \\
& \mathcal{D}_{0}=\{X \in \mathcal{C}: \varphi(X)=0\} .
\end{aligned}
$$

Recall the last condition ensuring convergence in the statement of lemma 3.1, namely: If $T\left(\mathcal{D}_{0} \backslash\{p\}\right) \subset \mathcal{D}_{+}^{\circ}$ then $\varphi(T(X)) \geq 0$ for $X \in \varphi^{-1}(0) \cap \mathcal{C}$ with equality only if $X=p$. Hence $\varphi \circ T$ is minimised on $\varphi^{-1}(0) \cap \mathcal{C}$ at $x=p \in \mathcal{C}$ which implies that there is a $\kappa \in \mathbb{R}$ such that $\kappa \nabla \varphi(p)+\nabla \varphi(p) D T(p)=0$. $\varphi$ defines a hypersurface $\mathcal{D}_{0}$ near $p$ we have $\nabla \varphi(p) \neq 0$ and hence $\nabla \varphi(p)$ is a left eigenvector of $D T(p)$ with eigenvalue $-\kappa$. But $D T(p)=I+D[p] D f(p)$ 
so that $-(1+\kappa) \nabla \varphi(p)=\nabla \varphi(p) D[p] D f(p)$. Comparison with $\nabla \varphi(p)=v^{\top} D f(p)$ suggests taking $v=D[p] \nabla \varphi(p)$. Setting $\alpha=\nabla \varphi(p)$ gives $v=D[p] \alpha$.

When $D T(p)^{-1} \gg 0$, which is the case for strongly competitive maps $T$, and also one of our assumptions (assumption 3), it is guaranteed by the Frobenius-Perron theorem for positive matrices that $v \gg 0$.

For the choice $v=D[p] \alpha$ we define $V, \psi, \varphi$ as above. The aim is to apply forward invariance theorems with $\mathcal{D}=\mathcal{D}_{+}$to establish that $\overline{\mathcal{D}_{+}}$is forward invariant. First, for Sacker's theorem, we require (i) $\mathcal{D}$ to be bounded and $T$ to be an open map on $\mathcal{D}_{+}^{\circ}$. This will be so if $T$ is an injective map of $\mathcal{D}_{+}^{\circ}$ onto $T \mathcal{D}_{+}^{\circ}$. Next (ii) the set $\mathcal{C} \backslash \overline{\mathcal{D}_{+}}$, i.e. $\mathcal{D}_{-}$, must have no bounded components. Finally, perhaps the most difficult part, we must show that (iii) $T\left(\partial \mathcal{D}_{+}\right) \subset \overline{\mathcal{D}_{+}}$. If (i)-(iii) hold true then by theorem 2.1 the set $\overline{\mathcal{D}_{+}}$is forward invariant.

Theorem 4.1. Under the assumptions A1-A3 for system (1), let $p$ be the unique interior fixed point of $T$ and $\alpha$ a positive left eigenvector of $D T(p)$. Define the function $\varphi: \mathcal{C} \rightarrow \mathbb{R}$ by $\varphi(X):=\alpha^{\top} D[p] \ln f(X)$ and the sets $\mathcal{D}_{+}, \mathcal{D}_{-}, \mathcal{D}_{0}$ via $(6)$. Suppose that $(i) \overline{\mathcal{D}_{+}}$is bounded and $T$ is a local homeomorphism on $\mathcal{D}_{+}$, (ii) $\mathcal{D}_{-}$has no bounded components in $\mathcal{C}$, (iii) $T\left(\mathcal{D}_{0} \cup\left(\overline{\mathcal{D}_{+}} \cap \partial \mathcal{C}\right)\right) \subset \overline{\mathcal{D}_{+}}$, and (iv) $T$ has no invariant set in $\partial \mathcal{C} \cap \overline{\mathcal{D}_{-}}$.

Then for each $x \in \mathcal{C}, \omega(x) \subseteq \mathcal{D}_{0} \cap V^{-1}(c)$ for some $c>0$.

Moreover, if $T\left(\mathcal{D}_{0} \backslash\{p\}\right) \subset \mathcal{D}_{+}^{\circ}$ then $\omega(x)=\{p\}$ for all $x \in \mathcal{C}$.

Proof. Conditions (i) to (iii) show that $\overline{\mathcal{D}_{+}}$is forward invariant by Sacker's theorem 2.1. By existence of the absorbing set $\mathcal{K}$ we need only consider $x \in \stackrel{\circ}{\mathrm{C}} \cap \mathcal{K}$. Given any $x \in \mathcal{C} \cap \mathcal{K}$, there are two possibilities: (a) $x(t)=T^{t}(x) \notin \overline{\mathcal{D}_{+}}$for any $t \in \mathbb{N}$ or $(\mathrm{b}) x(\tau+t) \in \overline{\mathcal{D}_{+}}$for some $\tau \in \mathbb{N}$ and all $t \in \mathbb{N}$.

In case (b) we immediately have $\varphi(x(\tau+t)) \geq 0$ for $t \in \mathbb{N}$. Thus by lemma $3.1 \omega(x) \subset$ $\mathcal{D}_{0} \cap V^{-1}(c)$. In case (a) we have $\varphi(x(t))<0$ for all $t \in \mathbb{N}$, i.e. $x(t) \in \mathcal{D}_{-}$for all $t \in \mathbb{N}$. By lemma 3.1, $\omega(x) \subset \mathcal{D}_{0} \cap V^{-1}(c)$. Finally, if $T\left(\mathcal{D}_{0} \backslash\{p\}\right) \subset \mathcal{D}_{+}^{\circ}$, then since $\omega(x) \subseteq \mathcal{D}_{0}$ is an invariant set and $T\left(\mathcal{D}_{0} \backslash\{p\}\right) \cap D_{0}=\emptyset$, we must have $\omega(x)=\{p\}$.

Next, we obtain an alternative version of theorem 4.1 that does not rely on openness of the mapping $T$, but instead relies upon connectness of invariant sets via theorem 2.2.

Theorem 4.2. Under the assumptions A1-A3 for system (1), let $p$ be the unique interior fixed point of $T$ and $\alpha$ a positive left eigenvector of $D T(p)$. Define the function $\varphi: \mathcal{C} \rightarrow \mathbb{R}$ by $\varphi(X):=\alpha^{\top} D[p] \ln f(X)$, the sets $\mathcal{D}_{+}, \mathcal{D}_{-}, \mathcal{D}_{0}$ via $(6)$, and $\Omega=\mathcal{C} \cap \mathcal{D}_{+}$. Suppose that (i) $\Omega$ is connected, (ii) $T(\Omega) \cap \Omega \neq \emptyset$, (iii) for each $X \in \mathcal{D}_{0},\{y \in \Omega: T(y)=X\}=\emptyset$, and (iv) $T$ has no invariant set in $\partial \mathcal{C} \cap \overline{\mathcal{D}_{-}}$.

Then for each $x \in \mathcal{C}, \omega(x) \subseteq \mathcal{D}_{0} \cap V^{-1}(c)$ for some $c>0$. 
Moreover, if $T\left(\mathcal{D}_{0} \backslash\{p\}\right) \subset \mathcal{D}_{+}^{\circ}$ then $\omega(x)=\{p\}$ for all $x \in \dot{\mathcal{C}}$.

Proof. By conditions (i)-(iii) and theorem $2.2, \bar{\Omega}=\overline{\mathcal{D}_{+}}$is forward invariant. The rest of the proof is similar to that of theorem 4.1.

The condition (iii) in theorem 4.1 can often be difficult to verify and it is useful to have an alternative approach that replaces this condition with one which is geometrically simpler and easier to verify.

The conditions of the following theorem that uses a tangent hyperplane approximation are sometimes easier to verify (as demonstrated in Example 5.1). We recall that a function $g$ from a convex subset of $\mathbb{R}^{N}$ to $\mathbb{R}$ is said to be convex if for any $x \neq y$ and $t \in(0,1)$ we have $g(t x+(1-t) y) \leq t g(x)+(1-t) g(y)$.

Theorem 4.3. Under the assumptions A1-A3 for system (1), let $p$ be the unique interior fixed point of $T$ and $\alpha$ a positive left eigenvector of $D T(p)$, and assume the function $\varphi(X)=$ $\alpha^{\top} D[p] \ln f(X)$ is convex and $\mathcal{D}_{-}$defined by (6) is unbounded. Let $h(X)=\alpha^{\top}(X-p)$ and $\mathcal{H}_{-}=\{X \in \mathcal{C}: h(X) \leq 0\}$. Then if (a) $T\left(\mathcal{H}_{-}\right) \subset \mathcal{H}_{-}$and (b) $h(T(X))<h(X)$ for all $X \in \mathcal{C} \backslash\{p\}$ with $h(X) \geq 0$, the fixed point $p$ globally attracts $\mathcal{C}$.

Proof. We first claim that $\omega(x) \subset \mathcal{H}_{-}$for all $x \in \mathcal{C}$. For $x \in \mathcal{H}_{-}$this is immediate by (a). For $x \in \mathcal{C} \backslash \mathcal{H}_{-}$, we have $h(x)>0$ so from (b) $h(T(x))<h(x)$. If there is an integer $m>0$ such that $h\left(T^{m}(x)\right) \leq 0$, then $T^{m}(x) \in \mathcal{H}_{-}$and the positive invariance of $\mathcal{H}_{-}$ implies that $T^{k}(x) \in \mathcal{H}_{-}$for all $k \geq m$ so $\omega(x) \subset \mathcal{H}_{-}$. Otherwise, we have $h\left(T^{m}(x)\right)>0$ for all $m>0$ so that $h\left(T^{m}(x)\right)$ is decreasing by (b). In this case there is a $c \geq 0$ such that $\lim _{m \rightarrow \infty} h\left(T^{m}(x)\right)=c$, i.e. $\omega(x) \subset h^{-1}(c)$. If $c>0$ then for any $y \in \omega(x)$, we have $h(y)=c>0$ so $h(T(y))<h(y)=c$. But the invariance of $\omega(x)$ implies that $T(y) \in \omega(x)$ so $h(T(y))=h(\omega(x))=c$, a contradiction. Therefore, $c=0$ so $\omega(x) \subset h^{-1}(0) \subset \mathcal{H}_{-}$.

Next, we show that $\omega(x)=\{p\}$ for all $x \in \mathcal{C}$. Suppose $x \in \mathcal{C}$ and $\omega(x) \backslash\{p\} \neq \emptyset$. Taking $y \in \omega(x) \backslash\{p\} \subset \mathcal{H}_{-}$, we have $h(y) \leq 0$ so either $h(y)<0$ or $h(T(y))<h(y)=0$. As both $y$ and $T(y)$ are in $\omega(x)$, we can always find an integer $m>0$ such that $T^{m}(x)$ is close enough to $y$ or $T(y)$ so that $h\left(T^{m}(x)\right) \leq 0$, i.e. $T^{m}(x) \in \mathcal{H}_{-}$. By the positive invariance of $\mathcal{H}_{-}$we have $T^{k}(x) \in \mathcal{H}_{-}$for all $k \geq m$. Since the function $\varphi(x)$ is convex, the set $\overline{\mathcal{D}_{-}}=\mathcal{D}_{-} \cup \mathcal{D}_{0}$ as defined through (6) is also convex. As $\mathcal{C} \cap h^{-1}(0)$ is tangent to $\mathcal{D}_{0}$ at $p, \overline{\mathcal{D}} \backslash\{p\}$ must be completely on one side of $\mathcal{C} \cap h^{-1}(0)$. Since $\mathcal{H}_{-}$is bounded but $\mathcal{D}_{-}$is unbounded, we must have $h(x)>0$ for all $x \in \overline{\mathcal{D}_{-}} \backslash\{p\}$. Therefore, $x \in \mathcal{H}_{-} \backslash\{p\}$ implies $x \in \mathcal{D}_{+}$, i.e. $\mathcal{H}_{-} \backslash\{p\} \subset \mathcal{D}_{+}$. Then, from lemma 3.1, we obtain $\omega(x) \subset \mathcal{H}_{-} \cap \mathcal{D}_{0}=\{p\}$, a contradiction to the supposition $\omega(x) \neq\{p\}$. Hence, $\omega(x)=\{p\}$ for all $x \in \mathcal{C}$.

Based on theorem 4.1, theorem 4.2 or theorem 4.3 , we are now in a position to deal with the global asymptotic stability of $p$, i.e. $\omega(x)=\{p\}$ for all $x \in \mathcal{C}$ and

$$
\forall \varepsilon>0, \exists \delta>0, \forall X \in \mathcal{B}_{\delta}(p) \cap \mathcal{C}, \forall t \geq 1, T^{t}(X) \in \mathcal{B}_{\varepsilon}(p) .
$$


(Here $\mathcal{B}_{r}(p)$ is the open ball centred at $p$ with a radius $r>0$.)

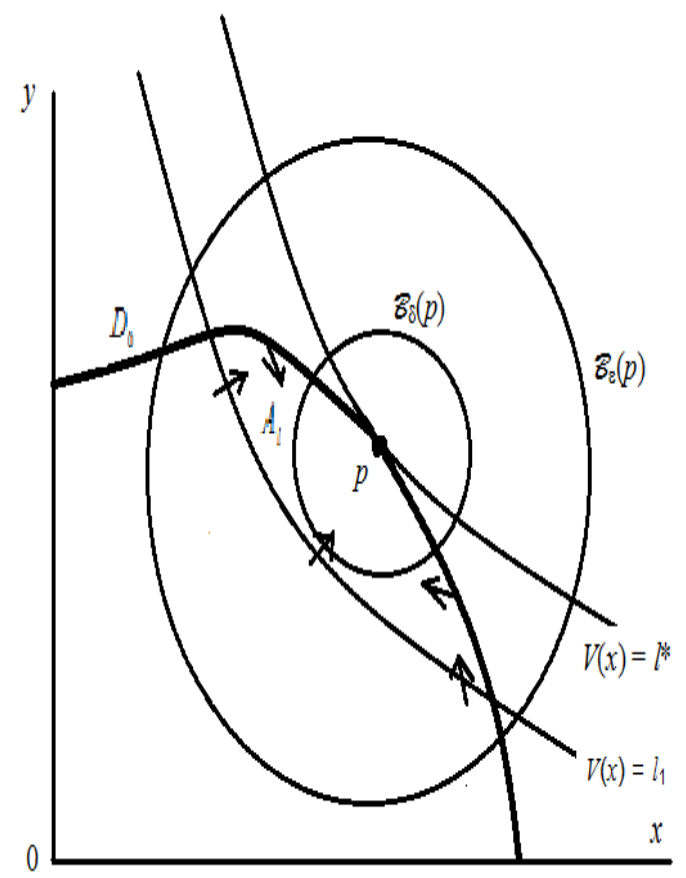

Figure 2. An illustration of the asymptotic stability argument in Theorem 4.4 of the interior fixed point $p$.

Theorem 4.4 (Split Lyapunov Stability). Assume that $p$ is globally attracting by theorem 4.1, theorem 4.2 or theorem 4.3. Then $p$ is globally asymptotically stable relative to $\mathcal{C}$ if one of the following conditions is met:

(a) Each eigenvalue $\mu$ of $D T(p)$ satisfies $|\mu|<1$.

(b) Under the conditions of theorem 4.1 or theorem 4.2, there exist $\rho>0$ and an integer $\tau>0$ such that $T^{\tau} \mathcal{B}_{\rho}(p) \subseteq \mathcal{D}_{+} \cup \mathcal{D}_{0}$.

(c) Under the conditions of theorem 4.3, there exist $\rho>0$ and an integer $\tau>0$ such that $T^{\tau} \mathcal{B}_{\rho}(p) \subseteq \mathcal{K}$, where $\mathcal{K}$ is the absorbing set of assumption $A 3$.

Proof. Under condition (a), $p$ is locally stable. Then the conclusion follows from this and the global attraction of $p$. 
Now suppose condition (b) holds. We need only show the local stability of $p$. Restricted to a small open ball centred at $p, \mathcal{D}_{0}$ and $V^{-1}(\ell) \cap \mathcal{\mathcal { C }}$ for any $\ell>0$ close to $V(p)$ are $(N-1)$ dimensional surfaces (see Figure 2). From the proof of theorem 4.1 or theorem 4.2 we know that $\overline{\mathcal{D}_{+}} \cap \mathcal{\mathcal { C }}$ is forward invariant. Thus, $x \in \overline{\mathcal{D}_{+}} \cap \mathcal{\mathcal { C }}$ implies $x(t)=T^{t}(x) \in \overline{\mathcal{D}_{+}} \cap \mathcal{\mathcal { C }}$ for all $t \in \mathbb{N}$ so that $V_{t+1}(x)=\psi_{t} V_{t}(x) \geq V_{t}(x), V_{t}(x)$ tends to $V(p)=\ell^{*}$ monotonically (since $x(t) \rightarrow p)$ as $t \rightarrow \infty$. If $x \in \overline{\mathcal{D}_{+}} \cap \mathcal{C}$ but $x \neq p$, the condition $T\left(\mathcal{D}_{0} \backslash\{p\}\right) \subset \mathcal{D}_{+}$implies that $V_{t}(x)$ strictly increases to $\ell^{*}$ as $t \rightarrow \infty$. This shows that $\mathcal{D}_{0} \cap V^{-1}\left(\ell^{*}\right) \cap \mathcal{C}=\{p\}$. Moreover, for any $\ell \in\left(0, \ell^{*}\right)$ close enough to $\ell^{*}$, the two surfaces $\mathcal{D}_{0}$ and $V^{-1}(\ell)$ intersect with each other near $p$ so that the closed set $A_{\ell}=\{X \in \mathcal{C}: \varphi(X) \geq 0, V(X) \geq \ell\}$ is forward invariant. Now for any given $\varepsilon>0$, we can choose $\ell \in\left(0, \ell^{*}\right)$ close enough to $\ell^{*}$ so that $A_{\ell} \subset \mathcal{B}_{\varepsilon}(p)$ (see Figure 2 ). Then, by (ii) and continuity of $T$, we can choose $\delta \in(0, \min \{\rho, \varepsilon\})$ sufficiently small such that

$$
\forall t \in I_{\tau}, T^{t} \mathcal{B}_{\delta}(p) \subset \mathcal{B}_{\varepsilon}(p) ; T^{\tau} \mathcal{B}_{\delta}(p) \subset A_{\ell}
$$

Hence, by forward invariance of $A_{\ell}$, we have $T^{t} \mathcal{B}_{\delta}(p) \subset \mathcal{B}_{\varepsilon}(p)$ for all $t=1,2, \ldots$ so $p$ is stable.

Suppose (c) holds. Then, since $(\mathcal{K} \cap \mathcal{C}) \subset\left(\mathcal{D}_{+} \cup\{p\}\right)$ and $T(\mathcal{K}) \subset \mathcal{K}$, the above reasoning under (b) is still valid after the replacement of $\overline{\mathcal{D}_{+}}$by $\mathcal{K}$.

4.1. Ultra-bounded population models. Some models of Kolmogorov form satisfy

$$
T_{k}(x)=x_{k} f_{k}(x) \rightarrow 0 \text { for each } k \text { as }|x|_{1} \rightarrow \infty \text { in } \mathcal{C} .
$$

(Here $\left.|x|_{1}:=\sum_{i=1}^{N}\left|x_{i}\right|\right)$.

Definition 4.5. We will call a map $T: \mathcal{C} \rightarrow \mathcal{C}$ satisfying (7) ultra-bounded.

Let $\Delta_{N}$ denote the $(N-1)$-dimensional unit probability simplex. Using simplex coordinates $R=|x|_{1}$ and $u_{k}=x_{k} / R$ we have that $T_{k}(R, u)=R u_{k} f_{k}(R u) \rightarrow 0$ as $R \rightarrow \infty$. For each $u \in \Delta_{N}$, the parameterised curve $\gamma_{u}=\{T(R, u): R \in[0, \infty)\}$ is a bounded and closed curve in $\mathcal{C}$. The set $T \mathcal{C}=\bigcup_{u \in \Delta} \gamma_{u}$ is, by construction, a compact absorbing set for $\mathcal{C}$. The boundary $\partial T \mathcal{C}$ of $T \mathcal{C}$ in $\mathcal{C}$ is the envelope of all the curves $\gamma_{u}$ as $u$ varies over $\Delta$ and for $X \in \partial T \mathcal{C}$ we have $\operatorname{det} D T(X)=0$. The map $T$ is $1-1$ restricted to $T \mathcal{C}$.

An example of an ultra-bounded model is the Ricker model $T_{k}(x)=x_{k} \exp \left(r_{k}\left(1-(\mathcal{A} x)_{k}\right)\right.$ where $r_{i}>0$ and the matrix $\mathcal{A} \geq 0$.

Thus we have the following result for ultra-bounded models.

Corollary 1. Under the assumptions A1-A3 for system (1), let $p$ be the unique interior fixed point of $T$ and $\alpha$ a positive left eigenvector of $D T(p)$. Define the function $\varphi: \mathcal{C} \rightarrow \mathbb{R}$ by $\varphi(X)=\alpha^{\top} D[p] \ln f(X)$ and the sets $\mathcal{D}_{+}, \mathcal{D}_{0}$ as in (6). Then $p$ is globally asymptotically stable if one of the following conditions holds:

(i) $T \mathcal{C} \subset \mathcal{D}_{+} \cup\{p\}$. 
(ii) $\mathcal{D}_{+} \cup\{p\} \subset T \mathcal{C}$ is absorbing for $\mathcal{C}$.

Proof. For either (i) or (ii) the conclusion follows directly from theorem 4.4.

\section{EXAmples}

For the two examples presented here local asymptotic stability of interior fixed points in these models (indeed their $N$-species versions) were studied by Roeger in a series of papers $[22,23,24]$. As far as we are aware, these global stability results obtained here are new.

Example 5.1 (3-species May-Leonard Leslie-Gower model)

Here

$$
T_{i}(x)=\frac{b x_{i}}{1+(\mathcal{A} x)_{i}}, i=1,2,3, \mathcal{A}=\left(\begin{array}{ccc}
1 & \alpha & \beta \\
\beta & 1 & \alpha \\
\alpha & \beta & 1
\end{array}\right), b, \alpha, \beta>0 .
$$

This is a strongly competitive map on $\mathcal{C}$.

Note that for $b<1$, the map $T$ is a contraction with $O$ as the unique fixed point. Thus we will assume $b>1$ in what follows.

The map $T$ has an unique interior fixed point at $p=\mu \mathbb{1}, \mu=(b-1) / \sigma$ where $\sigma=1+\alpha+\beta$. We will prove global asymptotic stability of $p$ in $\mathcal{C}$ (theorem 1.3 stated in section 1 ) via theorem 4.3. We recall that in theorem 4.3, we do not need to find how points on the curved hypersurface $\mathcal{D}_{0}$ move under the map $T$, which may often be a complex calculation, but rather we only need consider the movement under $T$ of points on certain hyperplanes.

From the definition of $T$ we obtain

$$
\begin{aligned}
D T(x)= & \operatorname{diag}\left[\frac{b}{1+(\mathcal{A} x)_{1}}, \frac{b}{1+(\mathcal{A} x)_{2}}, \frac{b}{1+(\mathcal{A} x)_{3}}\right] \\
& -\operatorname{diag}\left[\frac{b x_{1}}{\left(1+(\mathcal{A} x)_{1}\right)^{2}}, \frac{b x_{2}}{\left(1+(\mathcal{A} x)_{2}\right)^{2}}, \frac{b x_{3}}{\left(1+(\mathcal{A} x)_{3}\right)^{2}}\right] \mathcal{A} .
\end{aligned}
$$

It is known (for example, in [17]) that $T$ is injective on $\mathcal{C}$ and that (8) has a carrying simplex, but its geometry is not known.

$D T(p)$ has eigenvalues

$$
\lambda_{1}=\frac{1}{b}, \lambda_{2,3}=\frac{2 b(\alpha+\beta+1)+(b-1)(\alpha+\beta-2)}{2 b(\alpha+\beta+1)} \pm \frac{\sqrt{3}(b-1)|\alpha-\beta|}{2 b(\alpha+\beta+1)} i .
$$

Then $\left|\lambda_{2,3}\right|^{2}<1$ if and only if $\left(1-b^{-1}\right)\left[(\alpha-\beta)^{2}+(1-\alpha)(1-\beta)\right]<(\alpha+\beta+1)(2-\alpha-\beta)$. Under the conditions $b>1, \alpha \leq 1, \beta \leq 1$ with $\alpha+\beta<2$, we have

$(\alpha-\beta)^{2}+(1-\alpha)(1-\beta)-(\alpha+\beta+1)(2-\alpha-\beta)=2 \alpha(\alpha-1)+2 \beta(\beta-1)+\alpha \beta-1<0$. 
Thus, $p$ is asymptotically stable when $b>1, \alpha \leq 1, \beta \leq 1$ with $\alpha+\beta<2$. For larger values of $\alpha, \beta, p$ is stable provided that $b>1$ is large enough.

It remains to show by theorem 4.3 that $p$ attracts $\stackrel{\circ}{\mathcal{C}}$.

The matrix $D T(p)$ has a left eigenvector $\alpha^{\top}=(1,1,1)$ with associated eigenvalue $1 / b$. The function $\varphi(x)=\alpha^{\top} D[p] \ln f(x)$ is

$$
\varphi(x)=\frac{(b-1)}{\alpha+\beta+1}\left[3 \ln b-\ln \left(1+(\mathcal{A} x)_{1}\right)-\ln \left(1+(\mathcal{A} x)_{2}\right)-\ln \left(1+(\mathcal{A} x)_{3}\right)\right] .
$$

$\varphi(x)$ is convex, since it is the composition of orientation-preserving linear map and a convex map. Since $\varphi(x)<0$ for $\|x\|_{1}$ large enough, $\mathcal{D}_{-}$is unbounded. Define the function $\phi_{\theta}(x)=\theta-\|x\|_{1}$ for each $\theta \geq \theta_{0}=3(b-1) /(\alpha+\beta+1)$. Then $\Pi_{\theta}=\phi_{\theta}^{-1}(0)$ defines a plane with normal $(1,1,1)^{\top}$ passing through the point $\left(\frac{\theta}{3}, \frac{\theta}{3}, \frac{\theta}{3}\right)^{\top}$. We aim to show that for each $\theta \geq \theta_{0}, \phi_{\theta}(T(x))>0$ for all $x \in \Pi_{\theta} \cap \mathcal{C} \backslash\{p\}$. To this end we find the extrema of

$$
\phi_{\theta}(T(x))=\theta-\sum_{i=1}^{3} \frac{b x_{i}}{1+(\mathcal{A} x)_{i}}
$$

on $x \in \Pi_{\theta} \cap \mathcal{C}$. We need only show that for each $\theta \geq \theta_{0}, \phi_{\theta}(T(x))>0$, except $x=p$ when $\theta=\theta_{0}$ (under the conditions of theorem 1.3). But note that if for some $\theta_{1}>0$ and $x \in \mathcal{C}$ we have $\phi_{\theta_{1}}(T(x)) \geq 0$, then $\theta_{2}>\theta_{1}$ implies $\phi_{\theta_{2}}(T(x))>\phi_{\theta_{1}}(T(x)) \geq 0$. Hence we need only actually concern ourselves with the case $\theta=\theta_{0}$. From now on we drop the $\theta$ subscript on the understanding that $\theta=\theta_{0}$.

We now show that if $\phi(T(x))>0$ for $x \in \partial\left(\Pi_{\theta} \cap \mathcal{C}\right)$ then $\phi(T(x)) \geq 0$ for all $x \in \Pi_{\theta} \cap \mathcal{C}$ with equality only for $x=p$. Indeed, the set $\Pi_{\theta} \cap \mathcal{C}$ consists of line segments passing through $p$ with two ends in $\partial\left(\Pi_{\theta} \cap \mathcal{C}\right)$. Pick any such line segment with two points $q_{1}, q_{2} \in \partial\left(\Pi_{\theta} \cap \mathcal{C}\right)$ such that the line segment is given by $x(s)=s q_{1}+(1-s) q_{2} \in \Pi_{\theta} \cap \mathcal{C}$ for $s \in[0,1]$ and $x\left(s^{*}\right)=p$ for some $s^{*} \in(0,1)$. We need only show that $\phi(T(x(s)))>0$ for $s \in[0,1]$ with $s \neq s^{*}$. To this end, we note that $\phi(T(x(s)))$ vanishes if and only if $h(s)=H(x(s))$ vanishes where

$$
\begin{aligned}
H(x)= & \theta \prod_{i=1}^{3}\left(1+(\mathcal{A} x)_{i}\right)-b x_{1}\left(1+\left(\mathcal{A} x_{2}\right)\left(1+(\mathcal{A} x)_{3}\right)\right. \\
& -b x_{2}\left(1+(\mathcal{A} x)_{1}\right)\left(1+(\mathcal{A} x)_{3}\right)-b x_{3}\left(1+(\mathcal{A} x)_{1}\right)\left(1+(\mathcal{A} x)_{2}\right) .
\end{aligned}
$$

$h(s)$ is a polynomial of degree at most three in $s \in[0,1]$. Note that $h\left(s^{*}\right)=\theta b^{3}-b^{3}\|p\|_{1}=0$, and

$$
\begin{aligned}
h^{\prime}\left(s^{*}\right) & =\left(\theta-2 p_{1}\right) b^{2}(1,1,1) \mathcal{A}\left(q_{1}-q_{2}\right)-b^{3}(1,1,1)\left(q_{1}-q_{2}\right) \\
& =\left[b^{2} p_{1}(\alpha+\beta+1)-b^{3}\right]\left(\left\|q_{1}\right\|_{1}-\left\|q_{2}\right\|_{1}\right)=0 .
\end{aligned}
$$

Moreover, since by hypothesis $\phi(T(x))>0$ for $x \in \partial \Pi_{\theta} \cap \mathcal{C}$, we have $h(0)>0$ and $h(1)>0$. $h$ is a cubic satisfying $h(0)>0, h(1)>0, h\left(s^{*}\right)=0$ and $h^{\prime}\left(s^{*}\right)=0$ for some $s^{*} \in(0,1)$. 
Thus $s=s^{*}$ is a repeated root of $h$, and $h(s)>0$ for $s \in[0,1]$ with $s \neq s^{*}$. This shows that if $\phi \circ T$ is positive on the boundary $\partial\left(\Pi_{\theta} \cap \mathcal{C}\right)$ then it is positive on $\Pi_{\theta} \cap \mathcal{C} \backslash\{p\}$, which means that we need only consider $\phi \circ T$ on the boundary $\partial\left(\Pi_{\theta} \cap \mathcal{C}\right)$.

Now consider the new function

$$
\Psi_{\theta}(u)=\theta-\frac{b \theta u_{1}}{C u_{1}+A u_{2}+B u_{3}}-\frac{b \theta u_{2}}{B u_{1}+C u_{2}+A u_{3}}-\frac{b \theta u_{3}}{A u_{1}+B u_{2}+C u_{3}}
$$

on $u \in \Delta_{3}:=\left\{x \in \mathcal{C}:\|x\|_{1}=1\right\}$, where $C=\theta+1, A=\theta \alpha+1, B=\theta \beta+1$. Note that $\Psi_{\theta}(u) \geq 0$ on $\Delta_{3}$ if and only if $\phi_{\theta}(T(x)) \geq 0$ for $x \in \Pi_{\theta} \cap \mathcal{C}$.

The next step is to find conditions for which $\Psi(u)>0$ for $u \in \partial \Delta_{3}$.

For example, when $u_{3}=0$ we have

$$
\Psi\left(u_{1}, u_{2}, 0\right)=\theta-\frac{b \theta u_{1}}{C u_{1}+A u_{2}}-\frac{b \theta u_{2}}{B u_{1}+C u_{2}} .
$$

At the vertex $u=(1,0,0)^{\top}, \Psi(u)=\theta-\frac{b \theta}{C}>0$ if $b<C$. Similarly when $u=(0,1,0)^{\top}$, $\Psi(u)=\theta-\frac{b \theta}{C}>0$ when $b<C$. Thus $b<C$ is a necessary condition for $\Psi(u)>0$ on $\partial \Delta_{3}$. On a boundary line $u_{3}=0$ when $u_{2}>0$, we may introduce $X=u_{1} / u_{2}$ and consider the function

$$
p(X)=1-\frac{b X}{C X+A}-\frac{b}{B X+C} .
$$

Then $p(X)>0$ for all $X \geq 0$ if and only if $\Psi\left(u_{1}, u_{2}, 0\right)>0$ for $u_{1}=1-u_{2} \in[0,1]$.

Lemma 5.1. For $a_{1}, a_{2}, a_{3}, b_{1}, b_{2}, b_{4}, c_{1}>0$,

$$
c_{1}-\frac{a_{1} X}{a_{2} X+a_{3}}-\frac{b_{1}}{b_{2} X+b_{3}}>0, \quad \forall X \geq 0,
$$

when $a_{1} \leq a_{2} c_{1}, b_{1}<b_{3} c_{1}$ and either (i) or (ii) below holds:

(i) $\left(c_{1} a_{2} b_{3}+c_{1} a_{3} b_{2}-a_{1} b_{3}-b_{1} a_{2}\right)^{2}<4 a_{3} b_{2}\left(c_{1} a_{2}-a_{1}\right)\left(c_{1} b_{3}-b_{1}\right)$,

(ii) $a_{2} b_{1}+a_{1} b_{3}-a_{3} b_{2} c_{1}-a_{2} b_{3} c_{1} \leq 0$.

Proof. The expression on the left hand side of (9) can be written as

$$
\frac{X^{2}\left(a_{2} b_{2} c_{1}-a_{1} b_{2}\right)+X\left(a_{3} b_{2} c_{1}+a_{2} b_{3} c_{1}-a_{2} b_{1}-a_{1} b_{3}\right)+a_{3} b_{3} c_{1}-a_{3} b_{1}}{\left(a_{2} X+a_{3}\right)\left(b_{2} X+b_{3}\right)} .
$$

This is positive when

$$
X^{2}\left(a_{2} b_{2} c_{1}-a_{1} b_{2}\right)+X\left(a_{3} b_{2} c_{1}+a_{2} b_{3} c_{1}-a_{2} b_{1}-a_{1} b_{3}\right)+a_{3} b_{3} c_{1}-a_{3} b_{1}>0 .
$$

From the cases $X=0$ and $X>0$ large we require $a_{1} \leq a_{2} c_{1}$ and $b_{1}<b_{3} c_{1}$. If (ii) holds then the coefficients of $X$ and $X^{2}$ are nonnegative so the inequality holds for $X \geq 0$. Under (i) the polynomial has a positive minimum value. 
Applying lemma 5.1 to the function $p$ with $a_{1}=b, a_{2}=C, a_{3}=A, b_{1}=b, b_{2}=B$, $b_{3}=C, c_{1}=1$, we require $b<C$ and either $0<4 A B(C-b)^{2}-\left(C^{2}+A B-2 b C\right)^{2}=$ $\left(C^{2}-A B\right)\left(A B-(C-2 b)^{2}\right)=\left(C^{2}-A B\right)\left(4 b(C-b)-\left(C^{2}-A B\right)\right)$ or $2 b C-C^{2}-A B \leq 0$ for $\Psi>0$ on the boundary line $u_{3}=0$ of $\Delta_{3}$. The same conditions are needed on the other boundary lines. Note that $2 b C-A B-C^{2}<C^{2}-A B$ since $b<C$. Thus, $C^{2}-A B \leq 0$ implies $2 b C-A B-C^{2}<0$. In summary, we have

Lemma 5.2. The function $\phi(T(x))>0$ on the boundary of $\Pi_{\theta} \cap \mathcal{C}$ if and only if $C>b$ and at least one of the following inequalities holds:

$$
\begin{aligned}
\left(C^{2}-A B\right)\left(A B-(C-2 b)^{2}\right) & >0 \\
2 b C-C^{2}-A B & \leq 0 .
\end{aligned}
$$

First consider the condition $C>b$. This translates to $1+\theta_{0}>b$. Thus $\frac{3(b-1)}{1+\alpha+\beta}>b-1$ which requires

$$
\alpha+\beta<2 .
$$

Now

$$
\begin{aligned}
C^{2}-A B= & \left.\theta_{0}\left((2-\alpha-\beta)+(1-\alpha \beta) \theta_{0}\right)\right), \\
A B-(C-2 b)^{2}= & (b-1)(\alpha+\beta+1)^{-2}\left[3\left(\alpha^{2}+\beta^{2}-\alpha-\beta-\alpha \beta+1\right)\right. \\
& \left.-b\left(4 \alpha^{2}+4 \beta^{2}-4 \alpha-4 \beta-\alpha \beta+1\right)\right], \\
2 b C-C^{2}-A B= & -(b-1)(\alpha+\beta+1)^{-2}[3 b(1-2 \alpha-2 \beta+3 \alpha \beta) \\
& \left.-5(1-\alpha-\beta)+\alpha^{2}+\beta^{2}-7 \alpha \beta\right] .
\end{aligned}
$$

Thus when $\alpha+\beta<2$ we have $\alpha \beta<1$ and so $C^{2}>A B$. Hence when $\alpha+\beta<2$ and

$$
b\left(4 \alpha^{2}+4 \beta^{2}-4 \alpha-4 \beta-\alpha \beta+1\right)<3\left(\alpha^{2}+\beta^{2}-\alpha-\beta-\alpha \beta+1\right)
$$

inequality (10) of lemma 5.2 is satisfied. Similarly when $\alpha+\beta<2$ and

$$
3 b(1-2 \alpha-2 \beta+3 \alpha \beta) \geq 5(1-\alpha-\beta)+7 \alpha \beta-\alpha^{2}-\beta^{2}
$$

inequality (11) of lemma 5.2 is satisfied.

This establishes theorem 1.3 given in section 1 . Figure 3 (a) illustrates the region in $(b, \alpha, \beta)$ space satisfying the conditions of theorem 1.3 where the interior fixed point of Leslie-Gower model (8) is globally asymptotically stable. Figure 3 (b) shows the corresponding region where the interior point is (locally) asymptotically stable.

Example 5.2 (3-species May-Leonard Ricker model)

The Competitive Ricker Model is an analogue of the continuous time May-Leonard system for the Ricker model. The model has been studied by Hofbauer, Hutson and Jansen [13] in the context of permanence, and also by Roeger in terms of local asymptotic stability, 
(a)

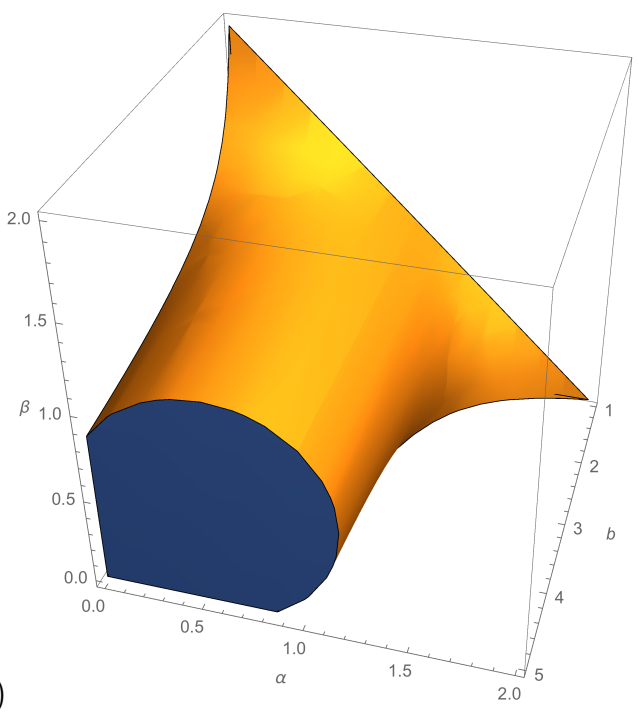

(b)

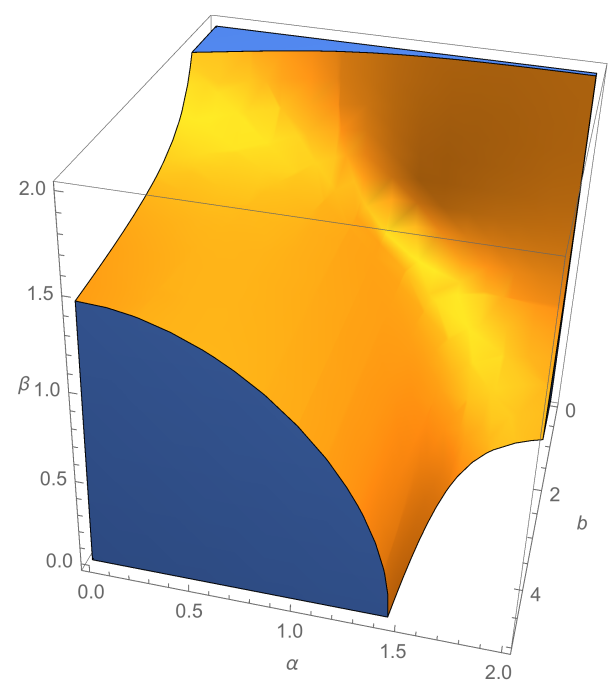

Figure 3. (a) Region in $(b, \alpha, \beta)$ space satisfying the conditions of theorem 1.3 where the interior fixed point of Leslie-Gower model (8) is globally asymptotically stable. (b) Region where the interior fixed point of LeslieGower model (8) is locally asymptotically stable.

limit cycles and heteroclinic cycles [22]. The map is defined by

$$
T_{i}(x)=x_{i} \exp r\left(1-(\mathcal{A} x)_{i}\right), \quad i \in I_{3}, \quad \mathcal{A}=\left(\begin{array}{ccc}
1 & \alpha & \beta \\
\beta & 1 & \alpha \\
\alpha & \beta & 1
\end{array}\right) .
$$

There is a unique interior fixed point $p$ that satisfies $\mathcal{A} p=\mathbb{1}=(1,1,1)^{\top}$, i.e. $p=\frac{1}{1+\alpha+\beta} \mathbb{1}$, if $(\alpha, \beta) \neq(1,1)$. It is clear that all orbits are bounded. Indeed the Ricker map is an ultra-bounded map.

We easily compute (see, for example, [23])

$$
D T(x)=\operatorname{diag}\left[e^{r-r(\mathcal{A} x)_{1}}, e^{r-r(\mathcal{A} x)_{2}}, e^{r-r(\mathcal{A} x)_{3}}\right](I-r D[x] \mathcal{A}) .
$$

At $x=p$ we have

$$
D T(p)=I-r \operatorname{diag}(p) \mathcal{A}=I-\frac{r}{1+\alpha+\beta} \mathcal{A} .
$$

Let $\lambda$ be an eigenvalue of $\mathcal{A}$. It is easy to see that $\mathcal{A}$ has a positive eigenvector $\alpha^{\top}=(1,1,1)$ and the corresponding eigenvalue is $\lambda=1+\alpha+\beta$. Hence $D T(p)$ has a positive (left) eigenvector $\alpha^{\top}=(1,1,1)$ associated with eigenvalue $\mu=1-r$. As shown in [23], $p$ is asymptotically stable when

$$
0<r<\max \left\{2,<\frac{(\alpha+\beta+1)}{\alpha^{2}+\beta^{2}-\alpha-\beta-\alpha \beta+1}\right\} .
$$


Clearly, det $D T(x)=0$ if and only if $\operatorname{det}(I-D[r x] \mathcal{A})=0$. If the matrix norm $\|D[r x] \mathcal{A}\|_{\infty}<$ 1 then $\operatorname{det}(I-D[r x] \mathcal{A}) \neq 0$ and $D T(x)^{-1} \gg 0$, so that $T$ is strongly competitive. Indeed in $\|x\|_{1}<1 / r$ we have $\|D[r x] \mathcal{A}\|_{\infty}<1$ provided that $\alpha, \beta<1$. This shows that $T(x)$ is strongly competitive and injective on $\left\{x \in \mathcal{C}: 0 \leq\|x\|_{1}<1 / r\right\}$ when $\alpha, \beta<1$.

The function $\varphi(x)=\alpha^{\top} D[p] \ln f(x)$ becomes $\varphi(x)=\frac{r}{\alpha+\beta+1} \sum_{j=1}^{3}\left(1-(\mathcal{A} x)_{j}\right)=\frac{r \alpha^{\top} \mathcal{A}(p-x)}{\alpha+\beta+1}=$ $r\left(\theta_{0}-\|x\|_{1}\right)$ where $\theta_{0}=3(1+\alpha+\beta)^{-1}$. For simplicity, we drop the factor $r$ from the expression of $\varphi$ without loss of generality. Then the sets $\mathcal{D}_{+}, \mathcal{D}_{0}, \mathcal{D}_{-}$are defined by $\varphi>0$, $\varphi=0, \varphi<0$ respectively. Clearly, the set $\mathcal{D}_{-}$is unbounded. The set $\mathcal{D}_{0}$ is a plane with normal direction $(1,1,1)$ passing through the interior fixed point $p=\frac{1}{1+\alpha+\beta} \mathbb{1}$.

That $T$ has no invariant set in $\partial \mathcal{C} \cap \overline{\mathcal{D}_{-}}$follows from the analysis of the two-dimensional Ricker model $[2,30]$.

Our task is now to derive conditions for $\varphi(T(x))>0$ for all $x \in \mathcal{D}_{0} \backslash\{p\}$. From $\varphi(x)=$ $\theta_{0}-x_{1}-x_{2}-x_{3}$ we have

$$
\begin{aligned}
\varphi(T(x)) & =\theta_{0}-x_{1} e^{r-r(\mathcal{A} x)_{1}}-x_{2} e^{r-r(\mathcal{A} x)_{2}}-x_{3} e^{r-r(\mathcal{A} x)_{3}} \\
& =\theta_{0}-\frac{x_{1}}{e^{-r+r(\mathcal{A} x)_{1}}}-\frac{x_{2}}{e^{-r+r(\mathcal{A} x)_{2}}}-\frac{x_{3}}{e^{-r+r(\mathcal{A} x)_{3}}} \\
& \geq \theta_{0}-\frac{x_{1}}{1-r+r(\mathcal{A} x)_{1}}-\frac{x_{2}}{1-r+r(\mathcal{A} x)_{2}}-\frac{x_{3}}{1-r+r(\mathcal{A} x)_{3}} .
\end{aligned}
$$

Set $\varrho(x)=\theta_{0}-\sum_{i=1}^{3} \frac{x_{i}}{1-r+r(\mathcal{A} x)_{i}}$. Then $\varphi(T(x))>0$ if we can show that $\varrho(x)>0$ for $x \in \mathcal{D}_{0} \backslash\{p\}$. Note that by introducing $b=1, C=r \theta_{0}+1-r, A=\alpha r \theta_{0}+1-r$ and $B=\beta r \theta_{0}+1-r$, we may reduce $\varrho$ in $u$ coordinates to the form of $\Psi$ in the previous example.

To apply lemma 5.2 to $\varrho$, we need to translate the conditions of inequalities (10) and (11) in terms of $r, \alpha, \beta$. The inequality $C>b$ translates to $r \theta_{0}+1-r>1$ which simplifies to $\alpha+\beta<2$ as in the previous example. Similarly we find that it is always the case that $C^{2}>A B$ and so that in addition to $\alpha+\beta<2$ we need either $A B-(C-2)^{2}>0$ or $A B-2 C+C^{2} \geq 0$. But $A B-(C-2)^{2}=\frac{r\left(2-(\alpha+\beta)^{2}+\alpha+\beta-3 r\left(\alpha^{2}-\alpha(\beta+1)+(\beta-1) \beta+1\right)\right)}{(\alpha+\beta+1)^{2}}$ and $A B-2 C+C^{2}=\frac{r\left(r\left(-\alpha^{2}-\alpha(5-7 \beta)-\beta(\beta+5)+5\right)-(\alpha+\beta+1)(2-\alpha-\beta)\right)}{(\alpha+\beta+1)^{2}}$. Hence we conclude that the Ricker model has a globally attracting interior fixed point when either

$$
3 r\left(1+\alpha^{2}+\beta^{2}-\alpha-\beta-\alpha \beta\right)<(2-\alpha-\beta)(1+\alpha+\beta)
$$

or

$$
r\left(5-\alpha^{2}-\beta^{2}-5(\alpha+\beta)+7 \alpha \beta\right) \geq(2-\alpha-\beta)(1+\alpha+\beta) .
$$

This completes the proof of Thorem 1.4.

Figure 4 (a) shows the regions in $(r, \alpha, \beta)$-space where global asymptotic stability for the Ricker model is obtained. Figure 4 (b) shows the corresponding region where the interior point is (locally) asymptotically stable. 
(a)

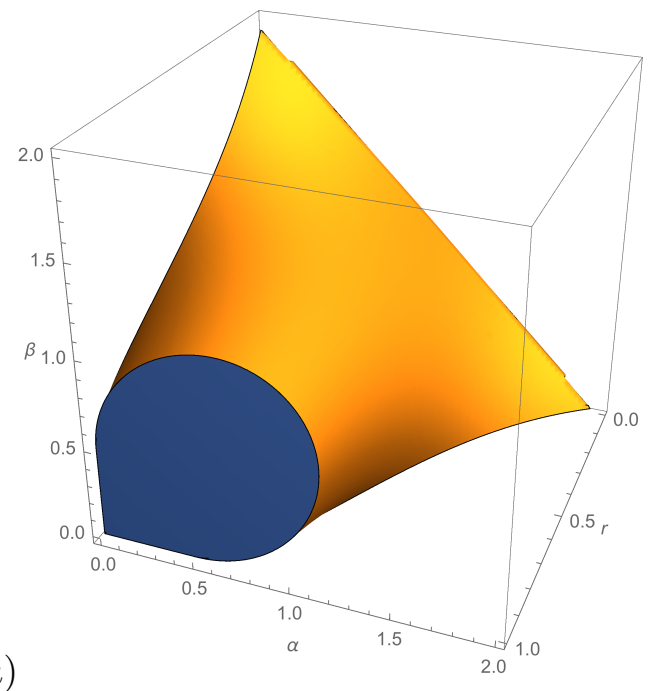

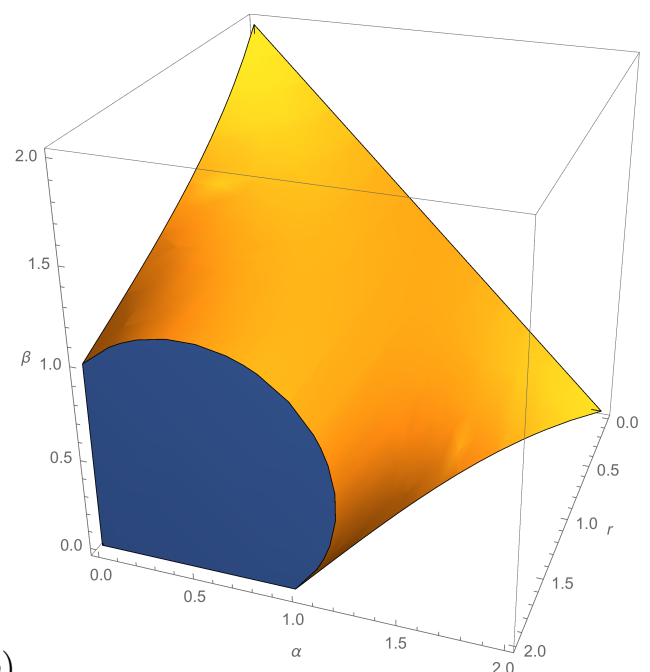

(b)

Figure 4. (a) Region in $(r, \alpha, \beta)$ space satisfying the conditions of theorem 1.4 where the Ricker model (12) is globally asymptotically stable. (b) Region where the Ricker model (12) is asymptotically stable. Note that in (a) the $r$ ranges $[0,1]$ whereas in (b) it ranges $[0,2]$.

\section{Conclusions}

Here we have described methods for identifying global asymptotic stability of interior fixed points of discrete-time population models and demonstrated their utility to derive new global stability results for 3 species competitive Leslie-Gower and Ricker models. We have attempted to elucidate our method in terms of ecological concepts, and in particular have introduced the idea of a principal reproductive mode, which is similar to Fisher's reproductive value, but not bound to age-structured models. We have also introduced the notion of the principal component of reproductive rate which measures the net reproductive growth using the principal reproductive mode. The eventual positiveness of the principal component of reproductive rate is diagnostic of global convergence to a steady coexistence state.

We have provided basic theorems for testing global attraction and stability of an interior fixed point in the first orthant, which is a generalisation of the split Lyapunov method from continuous dynamical systems to discrete systems. Our theory has been applied to 3 -species Leslie-Gower and Ricker models to provide new results concerning the regions of parameter space where the interior fixed point is globally asymptotically stable, and where previously only local stability was known. In a recent paper [16] the authors provide a partial phase portrait classification of the 3 -species Leslie-Gower model using index theory on the carrying simplex (a codimension-1 invariant manifold that attracts all points in 
the nonnegative cone bar the origin). Although we havenot made a full comparison of this method with ours, we note that our method does not require the identification of a carrying simplex.

\section{REFERENCES}

[1] S. Baigent and Z. Hou, Global stability of interior and boundary fixed points for Lotka-Volterra systems, Differential Equations Dynam. Systems, 20 (2012), pp. 53-66.

[2] J. M. Cushing, S. Levarge, N. Chitnis and S. M. Henson, Some discrete competition models and the competitive exclusion principle, J. Differ. Equ. Appl. 10 (2004), pp. 1139-1152.

[3] J. M. Cushing and Y. Zhou, The net reproductive value and stability in matrix population models, Nat. Resour. Model. 8 (1994), pp. 297-333.

[4] M. E. Fisher and B. S. Goh, Stability in a class of discrete time models of interacting populations, J. Math. Biol. 4 (1977), pp. 265-274.

[5] J. E. Franke and A. A. Yakubu, Global attractors in competitive systems, Nonlinear Anal. 16 (1991), pp. 111-129.

[6] J. E. Franke and A. A. Yakubu, Mutual exclusion versus coexistence for discrete competitive systems, J. Math. Biol. 30 (1991), pp. 161-168.

[7] J. E. Franke and A. A. Yakubu, Geometry of exclusion principles in discrete systems, J. Math. Anal. Appl. 168 (1992), pp. 385-400.

[8] J. E. Franke and A. A. Yakubu, Species extinction using geometry of level serfices, Nonlinear Anal. 21 (1993), pp. 369-378.

[9] J. E. Franke and A. A. Yakubu, Exclusion principles for density-dependent discrete pioneer-climax models, J. Math. Anal. Appl. 187 (1994), pp. 1019-1046.

[10] B. Garay and J. Hofbauer, Robust permanencefor ecological differential equations, minimax and discretizations, SIAM J. Math. Anal. 34 (2003), pp. 1007-1039.

[11] B. S. Goh, Global stability in many-species systems, Am. Nat. 111 (1977), pp. 135-143.

[12] J. Hofbauer, Saturated equilibria, permanence and stability for ecological systems, in Mathematical Ecology (Trieste, 1986), World Scientific, Teaneck, NJ, 1988, pp. 625-642.

[13] J. Hofbauer, V. Huston and W. Jansen, Coexistence for systems governed by difference equations of Lotka-Volterra type, J. Math. Biol. 25 (1987), pp. 553-570.

[14] Z. Hou and S. Baigent, Fixed point global attractors and repellors in competitive Lotka-Volterra systems, Dynam. Systems 26 (2011), pp. 367-390.

[15] Z. Hou and S. Baigent, Global stability and repulsion in autonomous Kolmogorov systems, Commun. Pure Appl. Anal. 14 (2015), pp. 1205-1238.

[16] J. Jiang and L. Niu, On the equivalent classification of three-dimensional competitive Leslie-Gower models via the boundary dynamics on the carrying simplex, J. Math. Biol. 74 (2016), pp. 1-39.

[17] J. Jiang, L. Niu and Y. Wang, On heteroclinic cycles of competitive maps via carrying simplices, J. Math. Biol. 72 (2015), pp. 1-34.

[18] R. Kon, Permanence of discrete-time Kolmogorov systems for two species and saturated fixed points, J. Math. Biol. 48 (2004), pp. 57-81.

[19] R. Kon, Convex dominates concave: An exclusion principle in discrete-time Kolmogorov systems, Proc. Amer. Math. Soc. 134 (2006), pp. 3025-3034.

[20] R. Kon and Y. Takeuchi, Effect of a parasitoid on permanence of competing hosts, Vietnam J. Math. 30 (2002), pp. 473-486.

[21] J. P. La Salle, The stability of dynamical systems, SIAM, CBMS-NSF Regional Conference Series in Applied Mathematics Vol. 25, SIAM, Philadelphia, PA, 1976.

[22] L. I. W. Roeger, Discrete May-Leonard competition models III, J. Differ. Equ. Appl. 10 (2004), pp. $773-790$. 
[23] L. I. W. Roeger, Discrete May-Leonard competition models II, Discrete Contin. Dyn. Syst. Ser. B 6 (2005), pp. 841-860.

[24] L. I. W. Roeger and L. J. S. Allen, Discrete May-Leonard competition models I, J. Differ. Equ. Appl. 10 (2004), pp. 77-98.

[25] A. Ruiz-Herrera, Exclusion and dominance in discrete population models via the carrying simplex, J. Differ. Equ. Appl. 19 (2013), pp. 96-113.

[26] R. J. Sacker, Global stability in a multi-species periodic Leslie-Gower model, J. Biol. Dyn. 5 (2011), pp. 549-562.

[27] R. J. Sacker, A note: An invariance theorem for mappings II, J. Differ. Equ. Appl. 18 (2012), pp. 595-599.

[28] R. J. Sacker and H. F. Von Bremen, Dynamic reduction with applications to mathematical biology and other areas, J. Biol. Dyn. 1 (2007), pp. 437-453.

[29] P. A. Samuelson, Generalizing Fisher's "reproductive value": linear differential and difference equations of "dilute" biological systems, Proc. Natl. Acad. Sci. USA 74 (1977), pp. 5189-5192.

[30] H. L. Smith, Planar competitive and cooperative difference equations, J. Differ. Equ. Appl. 3 (1998), pp. 335-357.

[31] Y. Takeuchi, Global dynamical properties of Lotka-Volterra systems, World Scientific, Singapore, 1996.

[32] E. C. Zeeman and M. L. Zeeman, From local to global behavior in competitive Lotka-Volterra systems, Trans. Amer. Math. Soc. 355 (2003), pp. 713-734.

E-mail address: s.baigent@ucl.ac.uk

E-mail address: z.hou@londonmet.ac.uk 